\title{
Chiral discrimination in biomimetic systems: Phenylalanine
}

\author{
K THIRUMOORTHY, K SONI, T ARUN and N NANDI* \\ Chemistry Group, Birla Institute of Technology and Science, Pilani, Rajasthan 333031 \\ e-mail: nnandi@bits-pilani.ac.in
}

MS received 1 June 2007; accepted 7 July 2007

\begin{abstract}
Chiral discrimination and recognition is important in peptide biosynthesis, amino acid synthesis and drug designing. Detailed structural information is available about the peptide synthesis in ribosome. However, no detailed study is available about the discrimination in peptide synthesis. We study the conformational energy variation of neutral methoxy phenyl alanine molecule as a function of its different dihedral angle to locate the minimum energy conformation using quantum chemical theory. We compared the intermolecular energy surfaces of phenyl alanine molecule in its neutral and zwitterionic state using quantum chemical theory as a function of distance and mutual orientation. The energy surfaces are studied with rigid geometry by varying the distance and orientation. The potential energy surfaces of $L-L$ and $D-L$ pairs are found to be dissimilar and reflect the underlying chirality of the homochiral pair and racemic nature of the heterochiral pair. The intermolecular energy surface of homochiral pair is more favourable than the corresponding energy surface of heterochiral pair.
\end{abstract}

Keywords. Peptide synthesis; ribosome; chiral discrimination; phenyl alanine; transition state.

\section{Introduction}

Biological chiral discrimination is an important phenomenon in amino acids or sugars where one enantiomeric form is exclusively selected by Nature during evolution over the other enantiomeric form. The Dsugar and L-amino acid are the relevant functional form rather than their mirror image isomers. While this discrimination is vital for function, the reason is unknown. The answer is rather difficult considering the identical physical properties (except those dependent on chirality) and closely similar energies of two isomeric forms of basic sub-units. Accurate retention of the biologically relevant chirality of basic sub-units like amino acids in protein remained a puzzle. How the homochirality composed of L-amino acids is retained during biosynthesis of a protein structure is an open question. Recent studies on biomimetic systems as monolayers and bilayers have shown that the differences in orientation dependent non-bonded and electrostatic interaction between enantiomeric and racemic pairs could lead to significant chiral discrimination. Development of different morphological features such as handedness of mesoscopic domains (in monolayers) or helicity (in

*For correspondence bilayers) is also well known. ${ }^{1-3}$ Theoretical calculation in model peptides indicates that dissymmetric arrangement of atoms or groups (as in a helical architecture) can lead to differences in interaction energy profile with an external non-bonded chiral ligand molecule leading to chiral discrimination. ${ }^{4}$

Further experimental evidence of observable chiral discrimination in biological systems is available in ribosomal peptide synthesis. ${ }^{5,6}$ Limited model theoretical analysis are carried out in this direction. ${ }^{7}$ How ribosome makes the discrimination possible remained unanswered and no quantitative analysis is available in this direction. Recent elegant and detailed crystal structure analysis corresponds to the natural (homochiral) peptide synthesis. For example, the crystal structure of CCA-Phe-cap-biotin bound simultaneously at half occupancy to both the A-site and P-site of the 50S ribosomal sub-unit ${ }^{8}$ is available which gives clear picture of the architecture suitable for peptide bond formation.

Recently, quantum mechanical study of the orientation-dependent energy surfaces of chiral and achiral amino acids showed that the non-bonded interaction between the amino acids could be significantly different for $L-L$ pair and $D-L$ pair. $^{7}$ As a process of mutual rotation of A-terminal and P-terminal leads to the peptide bond formation, it is likely that the 
chiral preference of A-site and P-site could be driven by the orientation dependent discrimination of amino acid segments. In order to understand the chiral discrimination in this system, a detailed molecular understanding of the conformational energy variation in Phenyl alanine moiety and the difference in the intermolecular interactions in $L-L$ and $D-L$ pairs is needed. To the best of our knowledge these studies are not carried out.

In a recent study the rotatory motion for $L-L$ and $D-L$ pair of phenyl alanine terminals are explored. ${ }^{9}$ While the rotatory motion of the $3^{\prime}$ end of the t-RNA for the $L-L$ pair do not encounter any repulsive interaction for the process of approach of A-terminal towards the P-terminal in the range of orientation suitable for the rotatory process. In contrast, the $D-$ $L$ pair could be strongly unfavourable within the ribosomal PTC that is suited for the corresponding rotatory motion of the natural $L-L$ pair. In this theoretical comparison, the surrounding of the terminals in the case of $D-L$ pair is not relaxed from the position of the same residues in the case of $L-L$ pair. Consequently, the energy gap observed in the calculation is the upper limit of discrimination. It is possible that the residues present in rabbit ribosomal PTC may re-arrange to accommodate the $D-L$ pair. However, no experimental data is available till date regarding the relaxation of the PTC. Of course, the ribosomal architecture is a dynamic one and the rearrangement is possible, which will bring the discrimination to a lowest limit when the two terminals will have identical energies (mirror image structures). Notably, discrimination may still arise from the possible difference in barrier heights in the reaction pathways of the $L-L$ and $D-L$ pair of reactants leading to corresponding peptides.

However, in order to ensure that the discrimination do not arise artificially from high energy conformational states of the terminals, it is important to note that the conformation of $\mathrm{D}$ - and L- are identical and their mutual configuration shall also be the minimum energy state. It is necessary to vary the mutual distance and orientation to validate the mutual configuration to be used in orientation dependent study.

With this end in view, we performed a detailed study of the conformational analysis of neutral and zwitterionic states of phenyl alanine. We also carry out a distance and orientation-dependent intermolecular energy surface. Finally, using the global minimum conformer, we carried out a transition state calculation to find out the differences in barrier heights. We describe the theoretical calculation in the following section.

\section{Theoretical calculation}

The crystal structure of CCA-Phe-cap-biotin bound simultaneously at half occupancy to both the A-site and P-site of the $50 \mathrm{~S}$ ribosomal sub-unit (PDB ID:1Q86) in the ribosomal part of Haloarcula marismortui is used to generate the L-conformer of phenyl alanine as observed in real system. ${ }^{8}$ An image of the peptidyl transferase cavity from the crystal structure of CCA-Phe-cap-biotin bound simultaneously at half occupancy to both the A-site and P-site of the $50 \mathrm{~S}$ ribosomal sub-unit taken from the crystal structure of the ribosomal part of the $H$. marismortui. (PDB ID: 1Q86) is shown in figure 1. Residues include (all numberings corresponds to scheme as in H. marismortui): A76 of CCA attached to phenyl alanine at A-terminal, A76 of CCA attached to phenyl alanine at P-terminal, A2485, A2486, C2487, A2488, U2620, A2637, G2540, U2451 and C2452. Enlarged views of A- and P-terminals in crystal structure of the ribosomal part of the $H$. marismortui. (PDB ID: 1Q86) are also shown. The carboxyl oxygen atom of the amino acid is linked to the tRNA. In our calculation, we studied both zwitterionic and neutral with carboxyl terminal as methoxy group in the conformational analysis.

In order to find the low energy conformation of Land D-phenyl alanine, the following variables are identified: dihedral angle related to carboxyl group, dihedral angle containing amine group and finally, dihedral angle containing $-\mathrm{CH}_{2} \mathrm{Ph}$ group. First, carboxyl rotation is varied and single point energies are calculated at HF/6-31G** level of theory. The lowest energy structure is used and amine group is varied at the same level of theory. Finally, the $\mathrm{CH}_{2} \mathrm{Ph}$ rotation is performed using the lowest energy conformer obtained from amine group rotation. A variation of $30^{\circ}$ increments are used throughout the conformational analysis. The results are given in figures 2 and 3 , for neutral states and zwitterionic respectively.

Starting from the geometry of A-site and P-site of the 50S ribosomal subunit (PDB ID:1Q86) in the ribosomal part of $H$. marismortui, mutual distance and orientation of the two phenyl alanine amino acids are varied. The results are presented in figures 4 and 5, respectively. This calculation will be useful to understand that whether the naturally available PTC environment is suitable for homo- or heterochiral 


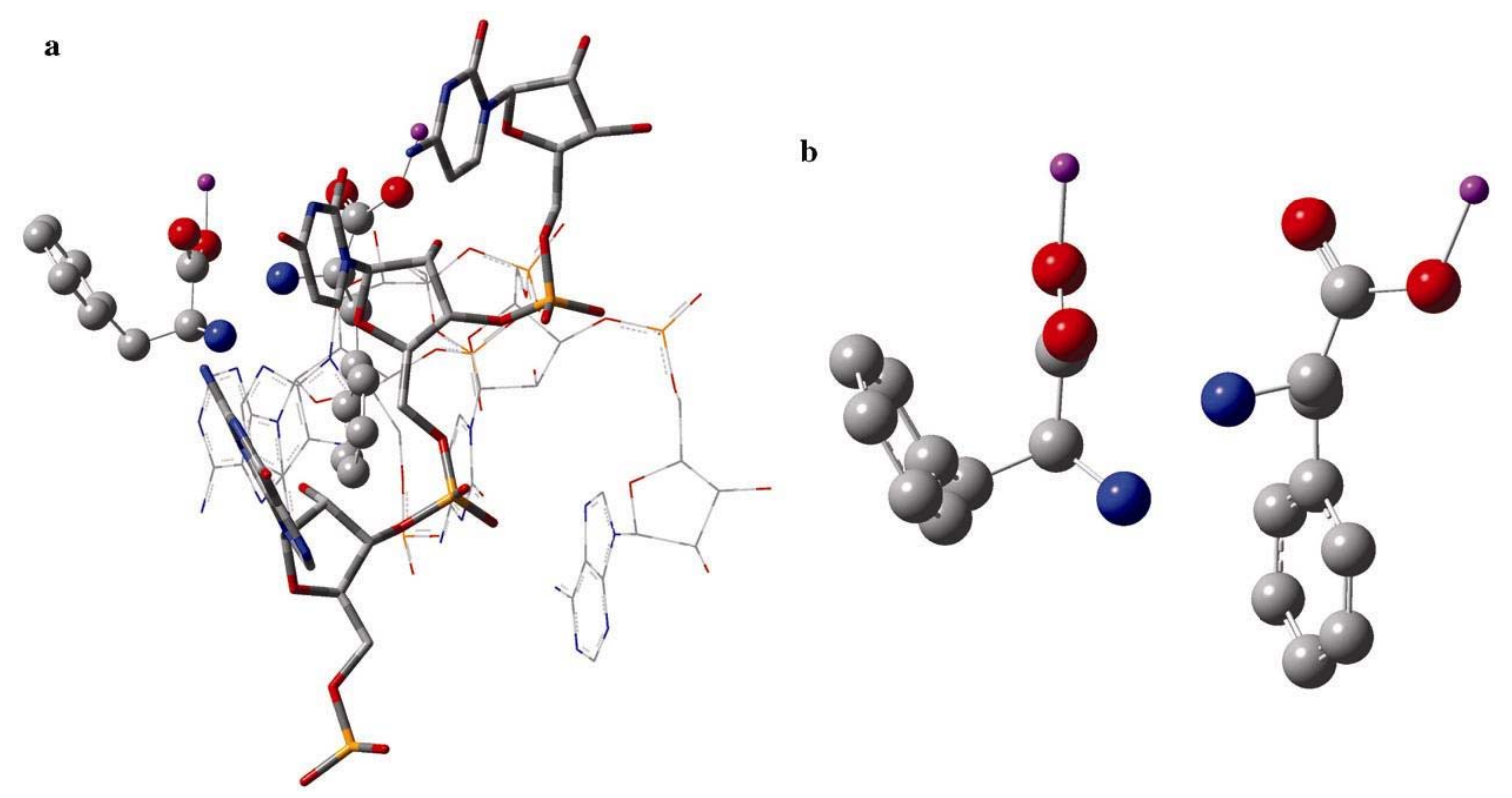

Figure 1. (a) An image of the peptidyl transferase cavity from the crystal structure of CCA-Phe-capbiotin bound simultaneously at half occupancy to both the A-site and P-site of the 50S ribosomal sub-unit taken from the crystal structure of the ribosomal part of the Haloarcula marismortui (PDB ID: 1Q86). ${ }^{8}$ Residues include (all numberings corresponds to scheme as in H. marismortui): A76 of CCA attached to phenyl alanine at A-terminal, A76 of CCA attached to phenyl alanine at P-terminal, A2485, A2486, C2487, A2488, U2620, A2637, G2540, U2451 and C2452, (b) enlarged view of A- and P-terminals in crystal structure of the ribosomal part of the H. Marismortui (PDB ID: 1Q86).

synthesis. This will give an upper limit of discrimination since, possibility exists that PTC containing heterochiral pair may undergo substantial structural re-arrangement and lower the repulsive interaction experienced by the D-terminal. However, no structural information regarding the re-arrangement is available to date.

Recently, in an experiment using the rabbit reticulocyte protein synthesis system, a series of puromycine derivatives were synthesized and stereoselectivity was measured using $\mathrm{IC}_{50}$ potency assay. ${ }^{6}$ Naturally occurring L-puromycine inhibits globin mRNA translation with an $\mathrm{IC}_{50}$ of $1.8 \mu \mathrm{M}$ and D-puromycine inhibit translation giving an $\mathrm{IC}_{50}$ of $280 \mu \mathrm{M}$. The difference is 150 -fold and is a signature of strong chiral discrimination. The experimentally observed discrimination is correlated with the difference in rate and hence, with the difference in the respective activation barrier of the corresponding homo- and heterochiral synthesis. In order to compare with this discrimination arising from difference in transition state barrier heights and concomitant rates of respective reactions, we computed the activation barrier of the transition state of $L-L$ and $D-L$ phenylalanine dipeptide at the HF/6-31G level of theory. Transition state calculations are performed with the neutral methoxy phenylalanine molecule at HF/6-31G level of theory. All calculations are performed using Gaussian suite of programs. ${ }^{10}$ In the next section, we present the results.

\section{Results and discussion}

As expected, the variation of conformational energy shown in the figures 2 and 3 for respective groups of L- and D-isomers are mirror images of each other. The minima in the carboxyl group rotation arise whenever the amine group is close to the carboxyl group. The two minima have the same energy in the case of neutral methoxy phenyl alanine as two hydrogens are approaching the carboxyl oxygen. The two minima are unequal in the case of zwitterionic structure. The carboxyl group rotation shows two maxima when the amine and carboxyl groups are apart. The two maxima are asymmetrical in the case of neutral methoxy phenyl alanine due to the asymmetry of $\mathrm{COOCH}_{3}$ group and symmetrical in the case of zwitterionic phenyl alanine due to the symmetry of the 
L-Phenylalanine: $\mathrm{COOCH}_{3}$ group rotation

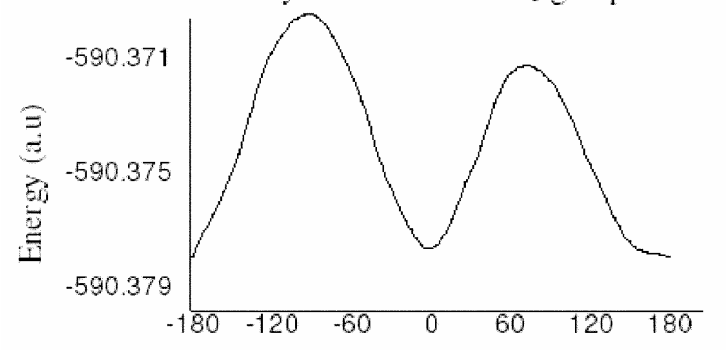

Dihedral angle (degree)

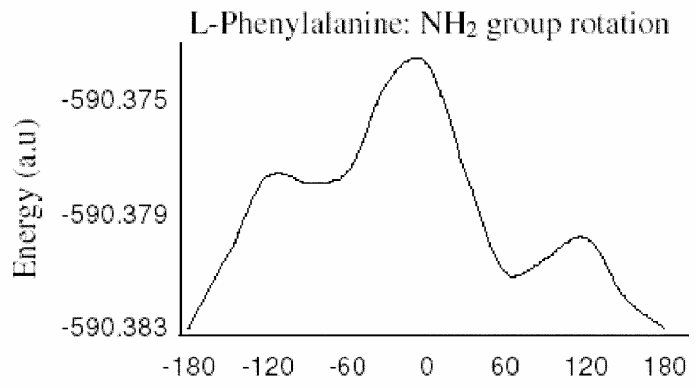

Dihedral angle (degree)

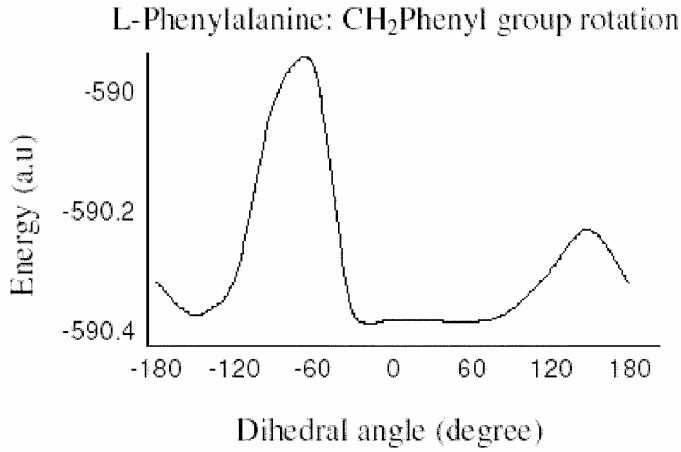

D-Phenylalanine: $\mathrm{COOCH}_{3}$ group rotation
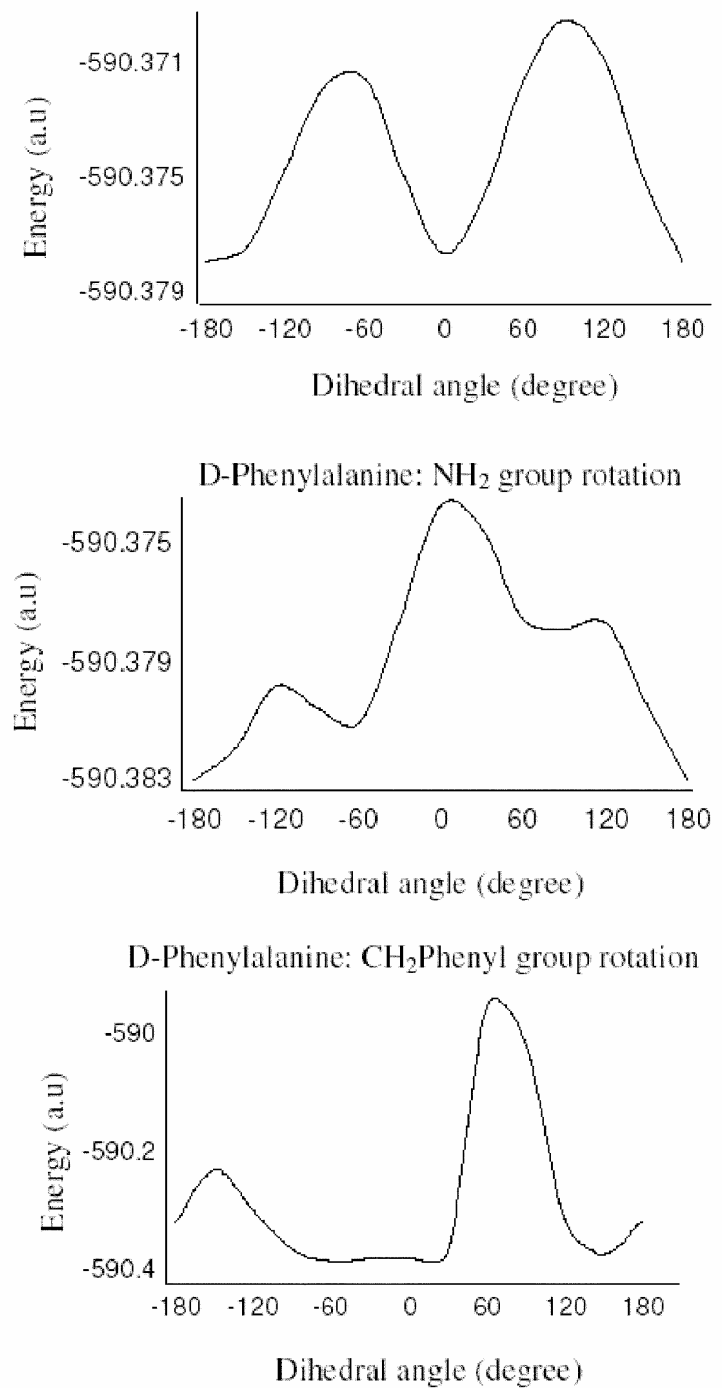

Figure 2. Variation in conformational energy as a function of rotation of $\mathrm{COOCH}_{3}, \mathrm{NH}_{2}$ and $\mathrm{CH}_{2} \mathrm{Ph}$ groups for neutral methoxy phenyl alanine. The related dihedral angles are $\mathrm{O}$ (of $\mathrm{COO}$ )-C (of $\mathrm{COO}$ )-C* (chiral carbon)-N(amine) for the rotation of $\mathrm{COOCH}_{3}, \mathrm{H}\left(\right.$ of $\left.\mathrm{NH}_{2}\right)-\mathrm{N}$ (of $\mathrm{NH}_{2}$ )-C* (chiral carbon)-C (of $\mathrm{CH}_{2}$ of $\mathrm{CH}_{2} \mathrm{Ph}$ ) for the rotation of $\mathrm{NH}_{2}, \mathrm{~N}$ (of $\left.\mathrm{NH}_{2}\right)-\mathrm{C}^{*}$ (chiral carbon)- $\mathrm{C}\left(\right.$ of $\mathrm{CH}_{2}$ of $\left.\mathrm{CH}_{2} \mathrm{Ph}\right)-\mathrm{C}(\mathrm{C}$ of $\mathrm{Ph}$ attached to $\mathrm{CH}_{2}$ ) for the rotation of $\mathrm{CH}_{2} \mathrm{Ph}$.

$\mathrm{COO}^{-}$group. The amine group rotation of neutral methoxy phenyl alanine shows minima when the amine hydrogens are close to the carboxyl group oxygen. The curve has different minima due to the different nature of electrostatic interaction with $\mathrm{CO}$ and $\mathrm{COCH}_{3}$ groups with amine hydrogens. On the other hand, the three-fold symmetry of $\mathrm{NH}_{3}^{+}$group is reflected in the periodic variation of minima at an interval of $120^{\circ}$. In the case of $\mathrm{CH}_{2} \mathrm{Ph}$ group, the higher maxima appear when the hydrogen of methylene group comes in close proximity of the carboxyl oxygen and the second maxima arise when the hydrogen of methylene group comes in close proximity of the amine group. Interestingly, the geometries of the two terminals are different from the minimum energy structures of either neutral methoxy phenyl alanine or zwitterionic structure. The geometries of A- and P-terminals themselves are also different. This is not surprising because, the low energy conformation of pair of molecules is expected to be different from 

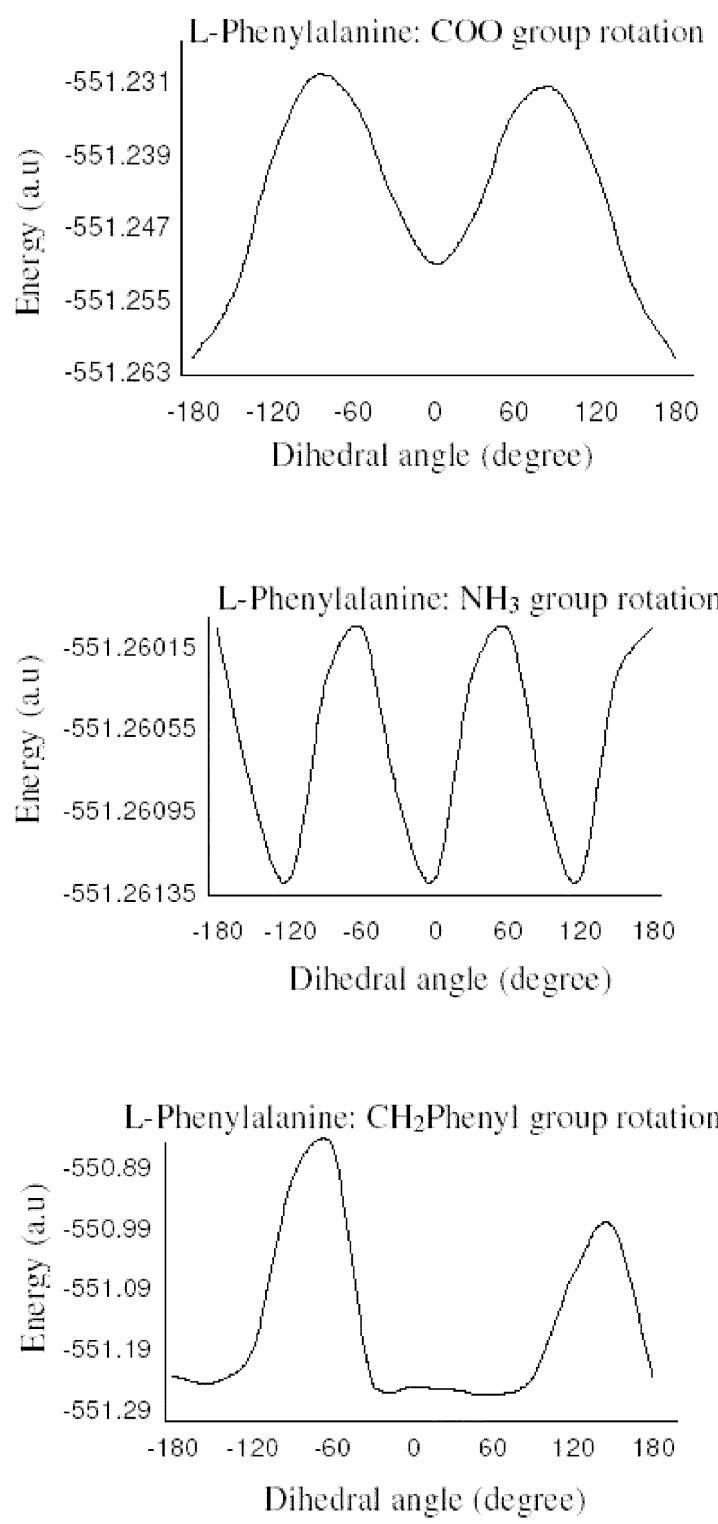

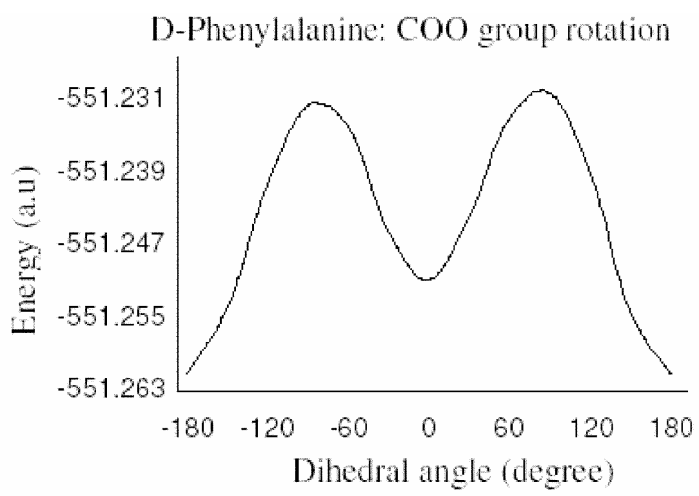

D-Phenylalanine: $\mathrm{NH}_{3}$ group rotation

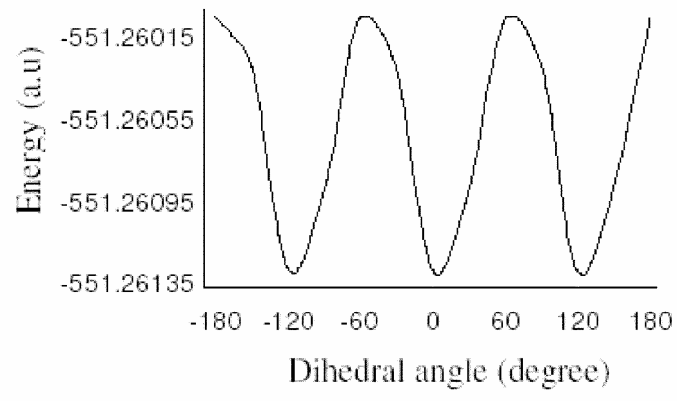

D-Phenylalanine: $\mathrm{CH}_{2}$ Phenyl group rotation

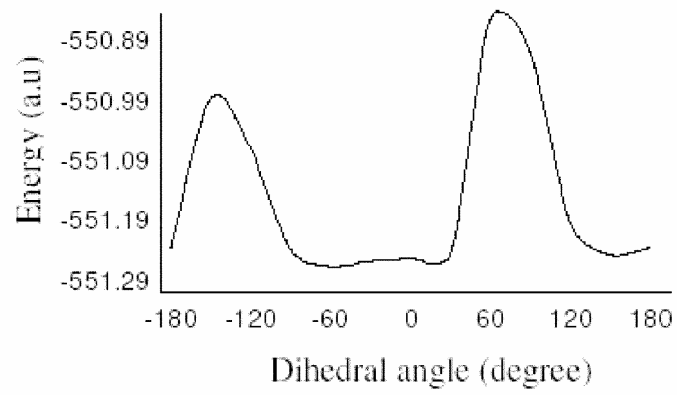

Figure 3. Variation in conformational energy as a function of rotation of $\mathrm{COO}-, \mathrm{NH}_{3}^{+}$and $\mathrm{CH}_{2} \mathrm{Ph}$ groups for zwitterionic phenyl alanine. The related dihedral angles are $\mathrm{O}\left(\right.$ of $\left.\mathrm{COO}^{-}\right)-\mathrm{C}$ (of $\left.\mathrm{COO}^{-}\right)-\mathrm{C}^{*}$ (chiral carbon)- $\mathrm{N}$ (amine) for the rotation of $\mathrm{COO}^{-}, \mathrm{H}$ (of $\left.\mathrm{NH}_{3}^{+}\right)-\mathrm{N}$ (of $\mathrm{NH}_{3}^{+}$)-C* (chiral carbon)-C (of $\mathrm{CH}_{2}$ of $\mathrm{CH}_{2} \mathrm{Ph}$ ) for the rotation of $\mathrm{NH}_{3}^{+}, \mathrm{N}$ (of $\left.\mathrm{NH}_{3}^{+}\right)-\mathrm{C}^{*}$ (chiral carbon)-C (of $\mathrm{CH}_{2}$ of $\left.\mathrm{CH}_{2} \mathrm{Ph}\right)-\mathrm{C}(\mathrm{C}$ of $\mathrm{Ph}$ attached to $\mathrm{CH}_{2}$ ) for the rotation of $\mathrm{CH}_{2} \mathrm{Ph}$.

the individual molecules and surrounding residues also influence the geometry of the molecules.

The change in the intermolecular energy of Aand P-terminals with the variation of mutual distance and orientation are presented in figures 4 and 5 , respectively for $L-L$ and $D-L$ pair. For this calculation, the chemical structures of terminals are mod- elled as follows: a methyl group replaces the connecting carbon atom at the t-RNA structure and the chemical structure is identical with neutral methoxy phenyl alanine. The results show that the pair configuration for $L-L$ and $D-L$ pair are indeed the minima. The energy rises as a function of distance and orientation for $L-L$ and $D-L$ pair. The energy rises more steeply 

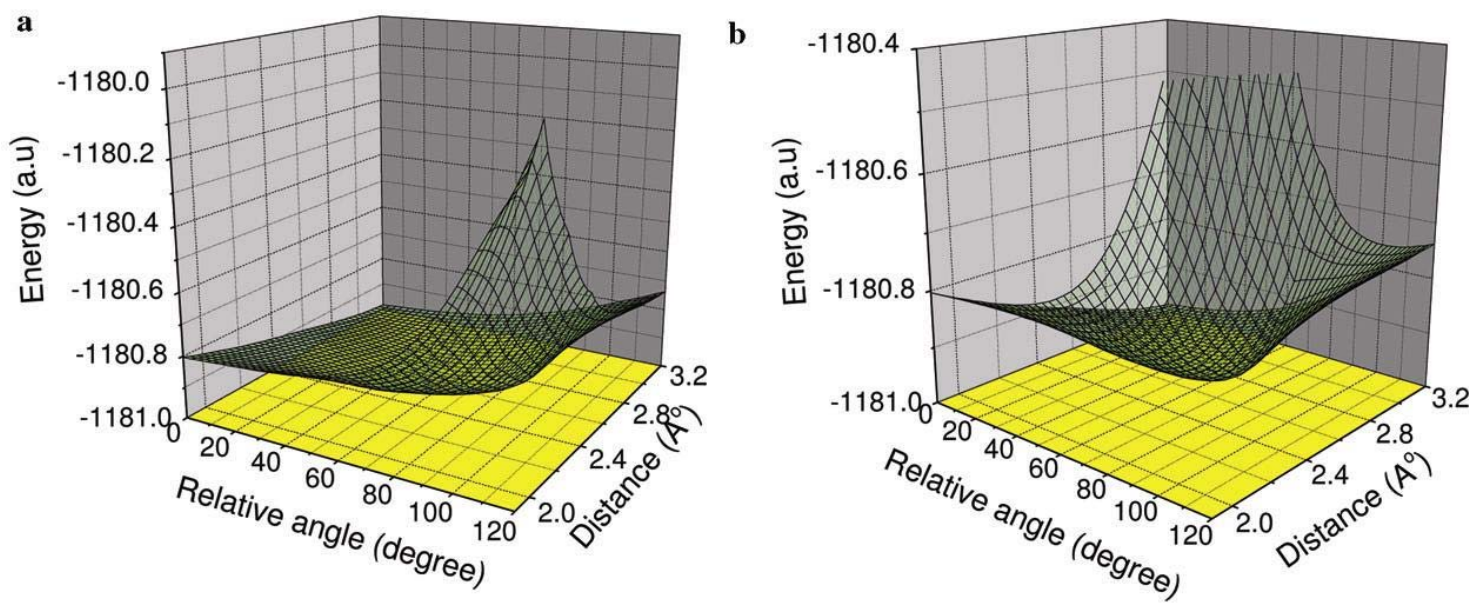

Figure 4. (a) Variation of intermolecular energy as a function of intermolecular distance and orientation of $L-L$ pair of A- and P-terminals in crystal structure of the ribosomal part of the H. marismortui. (PDB ID: 1Q86). The carbon atom attached to t-RNA part is replaced by a methyl group, (b) same plot with enlarged view of the minima region.
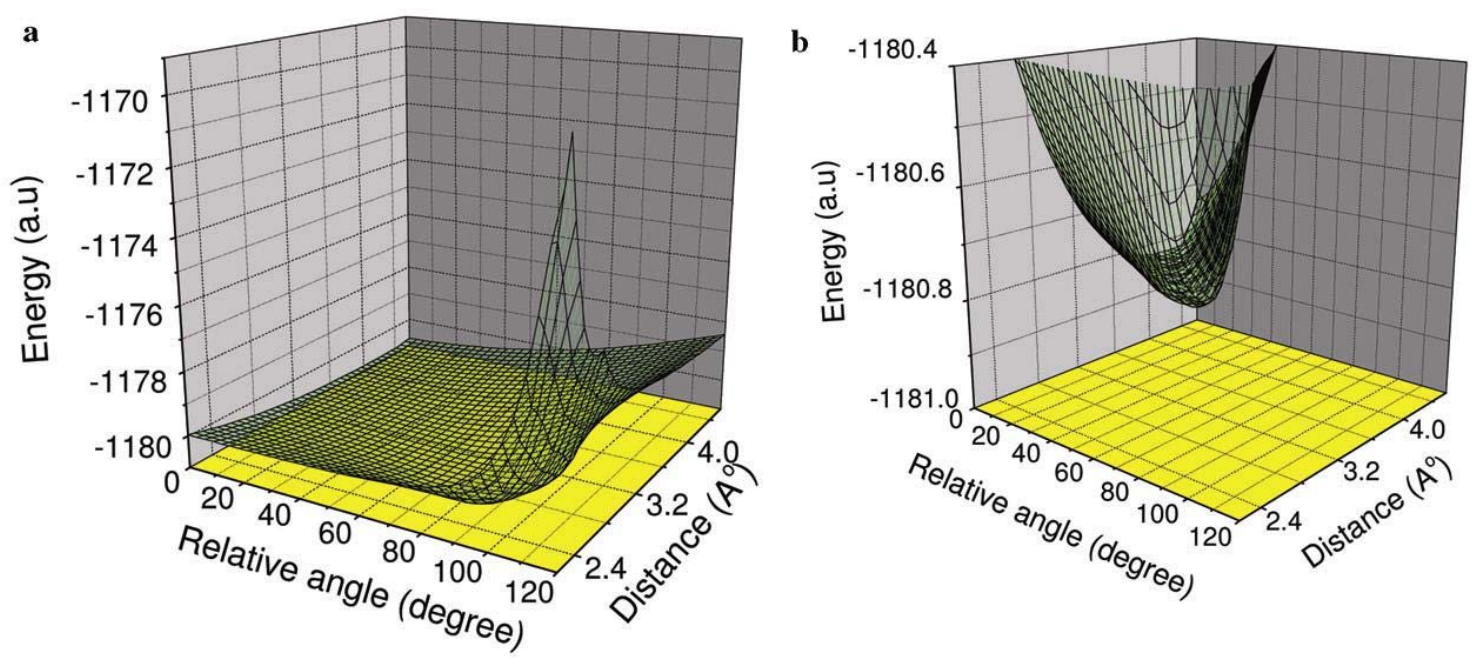

Figure 5. (a) Variation of intermolecular energy as a function of intermolecular distance and orientation of $D-L$ pair of A- and P-terminals in crystal structure of the ribosomal part of the H. marismortui. (PDB ID: 1Q86). The carbon atom attached to t-RNA part is replaced by a methyl group, (b) same plot with enlarged view of the minima region.

for $D-L$ pair than $L-L$ pair. This indicates that the rotatory space for $D-L$ pair is more restricted than $L-L$ pair. This is a signature of homochiral preference in the naturally available PTC. Notably, this discrimination is arising from phenyl alanine moieties themselves and not from the influence of surrounding residues. Non-bonded interactions between the side chains in the case of $L-L$ pair is less unfavourable than that in the case of $D-L$ pair.
The transition state barrier energy is calculated for both $L-L$ and $D-L$ neutral phenylalanine peptide bond formation reaction, which are shown in figure 6 . The differences in barrier height is $3.02 \mathrm{kcal} / \mathrm{mol}$ at the HF/6-31G level of theory. The corresponding rates are different and by the order of 164 . This also indicates that the rate of the $L-L$ dipeptide formation reaction is favourable than the rate of formation of $D-L$ pair and is a signature of homochiral preference. 


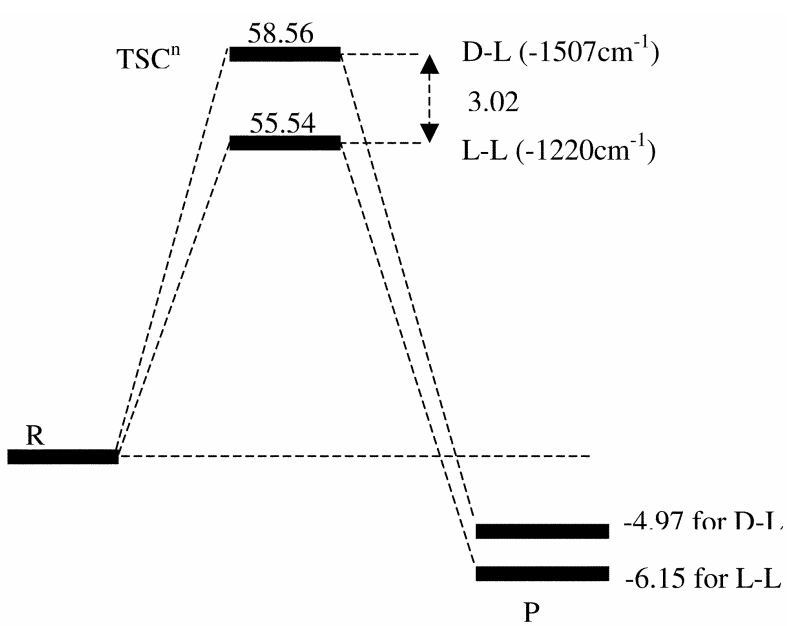

Figure 6. Comparison of the transition state energy level diagram of $L-L$ and $D-L$ phenylalanine peptide bond formation reaction at HF/6-31G level of theory. The relative energies are expressed in $\mathrm{kcal} / \mathrm{mol}$. The corresponding imaginary frequencies are noted as well as relative energies of transition states and products relative to the reactants.

\section{Conclusion}

We presented an analysis of the origin of the experimentally observed homochiral preference using quantum chemical method to a model of peptidyl transferase center based on the crystal structure of $H$. marismortui. The conformational analysis of phenyl alanine is performed and compared with the experimentally observed structure. The structures of the terminals are in a higher conformational energy state than the isolated molecule's global minimum state.
The rotational movement of A- and P-terminals in $L-L$ form is found to be energetically favoured over $D-L$ form, which indicates homochiral preference. The rate of the $L-L$ formation reaction is found to be favourable than the rate of formation of $D-L$ pair and is a signature of homochiral preference. The study provides an understanding that how homochirality is retained during peptide synthesis.

\section{Acknowledgement}

This work is sponsored by the Department of Science and Technology, India. Thanks are due to D Khetrapal, Chemistry Group, BITS, Pilani, for discussions.

\section{References}

1. Nandi N and Vollhardt D 2003 Chem. Rev. 1034033

2. Nandi N and Vollhardt D 2006 in Bottoms up nanofabrication: Supramolecules, self assemblies and organized films (ed.) K Ariga (American Scientific Publishers)

3. Nandi N and Vollhardt D 2007 Acc. Chem. Res. 40351

4. Nandi N 2004 J. Phys. Chem. B108 789

5. Tamura K and Schimmel P 2004 Science 3051253

6. Starck S R, Qi X, Olsen B N and Roberts R W 2003 J. Am. Chem. Soc. 1258090

7. Thirumoorthy K and Nandi N 2006 J. Phys. Chem. B110 8840

8. Hansen J L, Schmeing M T, Moore P B and Steitz T A 2002 Proc. Natl. Acad. Sci. (USA) 9911670

9. Thirumoorthy K and Nandi N 2007 J. Phys. Chem. B111 9999

10. Frisch M J et al 2004 Gaussian 03, Revision C.02 (Wallingford CT: Gaussian, Inc.) 